\title{
Behavior of Ad Hoc routing protocols, analyzed for emergency and rescue scenarios, on a real urban area \\ Liliana Enciso Quispe $^{\text {a,* }}$, Luis Mengual Galan ${ }^{b}$
}

\footnotetext{
${ }^{a}$ Research Institute of Computer Science, Technical University of Loja, San Cayetano alto, Loja, Ecuador

${ }^{\mathrm{b}}$ Department of Computing, Polytechnic University of Madrid, Boadilla del Monte, Madrid, Spain
}

\section{A B S T R A C T}

\begin{abstract}
A mobile Ad Hoc network (MANET) is a collection of wireless mobile nodes that can dynamically config-ure a network without a fixed infrastructure or central administration. This makes it ideal for emergency and rescue scenarios, where sharing information is essential and should occur as soon as possible. This article discusses which of the routing strategies for mobile MANETs: proactive, reactive or hierarchical, has a better performance in such scenarios. By selecting a real urban area for the emergency and rescue scenario, we calculated the density of nodes and the mobility model needed for the validation study of AODV, DSDV and CBRP in the routing model. The NS2 simulator has been used for our study. We also show that the hierarchical routing strategies are better suited for this type of scenarios.
\end{abstract}

\section{Introduction}

Mobile Ad Hoc Networks, are dynamically configurable wireless networks without fixed infrastructure or central administrative management. Their nodes can be the source, the destination, and the bridge of information. They have finite resources that should be well used with the aim of improving the performance of the entire network. Continuous improvement of the end-user hardware equipment (mobile devices and phones) in wireless network technologies helps to accelerate transmission speed. The rise of new applications has enabled more users to capitalize these smart devices. The nodes in the MANET, work using a set of elements denoted scenario. A scenario is composed of a specific number of nodes, topography and the definition of a mobility algorithm, which includes: direction, speed and pauses of the nodes. In case of Emergency and Rescue Scenarios (ERS), the topography is different in the amount of obstacles that may arise due to the occurrence of undesired events that cause the alteration of the normal mobility pathways; after this, new routes for evacuation or rescue should be calculated. Therefore, the algorithm of motion is determined according to the topography, and the corresponding nodes must be moved depending on the obstacles. The number of nodes depends on the MANET network and if it is in an urban or in a rural area. When MANET networks are used specifically in ERS, the choice of a robust network protocol is essential, because it involves the care and integrity of the person using the mobile device. If a device is disconnected from the network by battery discharge or

\footnotetext{
* Corresponding author. Tel.: +593983207252.

E-mail addresses: Ienciso@utpl.edu.ec (L.E. Quispe), Imengual@fi.upm.es (L.M. Galan).
}

malfunction, it must be temporarily removed from the routing list to reduce congestion.

According to the author (Ramrekha, Talooki, Rodriguez, \& Politis, 2012), the routing protocols in extreme emergency situations must be energy efficient and scalable. In (Thorsten Aurisch, 2009), the advantages of using an Ad Hoc network instead of a fixed network in emergency and rescue scenarios are compared. Here, the authors explain that a multi-hop route, such as an $\mathrm{Ad}$ Hoc network, allows better communication as soon as the emergency services reach the area in crisis and the links try to stay within the transmission range of the devices. Others have shown how is the behaviour of reactive protocols in emergency and disaster scenarios (Reina, Toral, Barrero, Bessis, \& Asimakopoulou, 2011, 2012). This analysis shows that among the reactive protocols, AODV provides the best results in terms of routing metrics within its category. There are also studies suggesting that DSDV could be the most suitable protocol for emergency and rescue scenario, since at any given time the required routing tables are updated to take the reference point in an evacuation (Sadasivam, Changrani, \& Yang, 2005). It is also mentioned that among the most common proactive protocols, DSDV performs better in terms of performance and packet delivery fraction (Kumawat \& Somani, 2011).

Given the importance of the issue, in emergency and rescue scenarios, other researchers (Gupta \& Saket, 2011; Jadeja \& Patel, 2013; Sadasivam et al., 2005) have made a comparative analysis between reactive and proactive protocols, and obviously determined that reactive routing protocols deal better with delays and routing overhead.

There are also other specific research protocols in emergency and rescue scenarios that have been analyzed, which have been performing well in the metrics overlap and delay for solving 
connectivity problems in disaster scenarios. (Fujiwara \& Watanabe, 2005; Chen, Pea-Mora, Plans, Mehta, \& Aziz, 2012; Reina, Marn, Bessis, Barrero, \& Asimakopoulou, 2013).

The main objective of this paper is to determine which one of the Ad Hoc routing strategies: proactive, reactive and hierarchical, performs better in emergency and rescue operations. We have chosen the urban area of the city of Loja, in Ecuador, to simulate these protocols, supported by the NS2 tool. NS2 is a discrete event network simulator that is used with wired and wireless networks.

\section{Related work}

The network layer (Ali, Ahmad, \& Aljunid, 2008), with respect to the OSI reference model, is where one performs and identifies the processes of MANETs. Hence, any improvement effort in this layer is directly visible in the upper layers. The routing protocols of MANETs are generally grouped into proactive, reactive and hierarchical routing (Overview \& Selangor, 2007).

In this article we pretend to comparatively evaluate three different types of routing protocols (proactive, reactive and hierarchical) in emergency and rescue scenarios to be applied in urban areas.

\subsection{Routing protocols in ERS}

Depending on their characteristics, we analyze each of them:

Proactive routing protocols maintain information on all routes throughout the network, even if they are not required, so each node registers routes to all other nodes in the network. These protocols exchange control information between nodes on a regular basis, which keeps updated routes for each node in the network. They also react when a new node appears or another node is no longer within the network topology. The most known proactive protocols are: Destination-Sequence Distance-Vector (DSDV) (Mahdipour, Rahmani, \& Aminian, 2009) and Optimized Link State Routing (OLSR) (Zhiyuan \& Jinhong, 2010). The idea of proactive routing is to distribute the information periodically through the network in order to pre-calculate all possible paths. When a change occurs, updates are propagated to keep routing tables refreshed. Therefore, in emergency and rescue scenarios, making a continuous assessment of the routes between nodes is very important to eventually evacuate people who are in the affected area. However, too much of these actions may cause overloading, which directly affects the utilization of bandwidth and energy efficiency. In a static topology, this routing scheme can work properly and, in large and highly dynamic networks, non-management has a good scalability.

Reactive routing protocols allow update of the tables on demand. For example, when a particular node wants to exchange information in the network. They usually have two components: route discovery, which occurs when a node wants to communicate with a specific destination, and route maintenance, used to manage the path failure caused by the mobility of the nodes. The route discovery ends when we discover the path to the destination node or when all alternatives have been sought without finding any route. The difficulty with these protocols is the latency to initiate communications; they also have a slower reaction to detect changes in the network topology. The advantage of using them, in emergency and rescue scenarios, would be energy savings during communications, since a non-constant network update improves energy saving on mobile devices from people waiting for a communication link to address the evacuation zone. Among the most known reactive protocols are: Dynamic Source Routing (DSR) (Johnson, Hu, \& Maltz, 2007) and Ad Hoc Demand Distance Vector (AODV) (Shukla, Jha, Saxena, \& Biswash, 2013).
Hierarchical routing protocols divide the network into subsets of nodes called clusters, where a cluster head node is used to concentrate and distribute the information generated within the group. An example of this type of protocol is the Cluster Based Routing Protocol (CBRP) (Chaba, Singh, \& Joon, 2009). Fig. 1 shows the basic components of a hierarchical routing protocol or cluster. The advantage of using this type of routing, in emergency and rescue scenarios, is in the administration of the network through a cluster head for each cluster. This kind of grouping puts order in peoples evacuation and thus, communication between nodes is faster, considering that now communications are no longer across the entire network, but locally. With this, we save energy, bandwidth, and network performance is also better.

There are some studies (Mahesh Motwani \& Agarwal, 2009; Biradar \& Patil, 2006), that identify and group the hierarchical routing algorithms or clustering. These protocols and hierarchical routing strategies focus on the task of choosing the cluster head and on cluster maintenance. For example, Er and Seah $(2004,2005)$ focus on the choice of cluster based solely on the property of the node mobility. In turn, Chaba et al. (2009), Gerla95multicluster,amis; perform cluster head election used as the deciding factor node identification. Zang and Tao (2009) and Torres et al. (2012) uses the distance between nodes or the degree of connectivity for the election. McDonald and Znati (1999) makes the choice of cluster head periodically in order to save energy. The protocols proposed in Er and Seah (2004) and Jahani and Bagherpour (2011), made the choice of cluster head based on the combined weights of each node characteristics.

As we can see, there is little research information about hierarchical protocol simulation for emergency and rescue scenarios. Most researchers are delving into the analysis of Clusters and Cluster Heads, considering that they are relevant for finding optimal solutions and for successfully manage network resources. For this reason, we made use of the advantages of this routing type and applied it to respective scenarios.

As shown in previous researches, reactive protocols present advantages over proactive protocols. Now, our choice of the routing protocols: CBRP, AODV and DSDV, was taken under the following considerations:

CBRP is a reactive protocol in general terms (Ugas, 2009), but it also has a hierarchical component due to its level-oriented administration and because each cluster is governed by a cluster head. With this type of routing for Ad Hoc networking development, there is a significant advantage in energy consumption metrics, bandwidth and network performance (Jahani \& Bagherpour, 2011). However, it is important to note that there is little information and research on this type of routing for emergency and rescue situations. Obviously, this was one of the considerations for choosing CBRP. On the other hand, AODV (reactive routing protocol) and DSDV (proactive routing protocol) were chosen because in their category, they showed the best performance. (Reina, Toral, Barrero, Bessis, \& Asimakopoulou, 2012; Kumawat \& Somani, 2011).

\subsection{Mobility models for MANETs in ERS}

Mobility models are important because they determine the behavior of mobile nodes (MN) (Camp, Boleng, \& Davies, 2002; Niewiadomska-Szynkiewicz, Sikora, \& KoAodziej, 2013). They can be classified into two types: those based on traces (logs of actual movements) and the synthetic (emulate reality by mathematical equations). Other authors classify mobility models into three groups (Camp et al., 2002): models based on strokes (work with real mobility), models based on topology restrictions (real scenario simulations) and statistical models (study from randomness). MANETs do not work yet on models based on traces in the network characteristics. But certainly, it is expected that the study and 


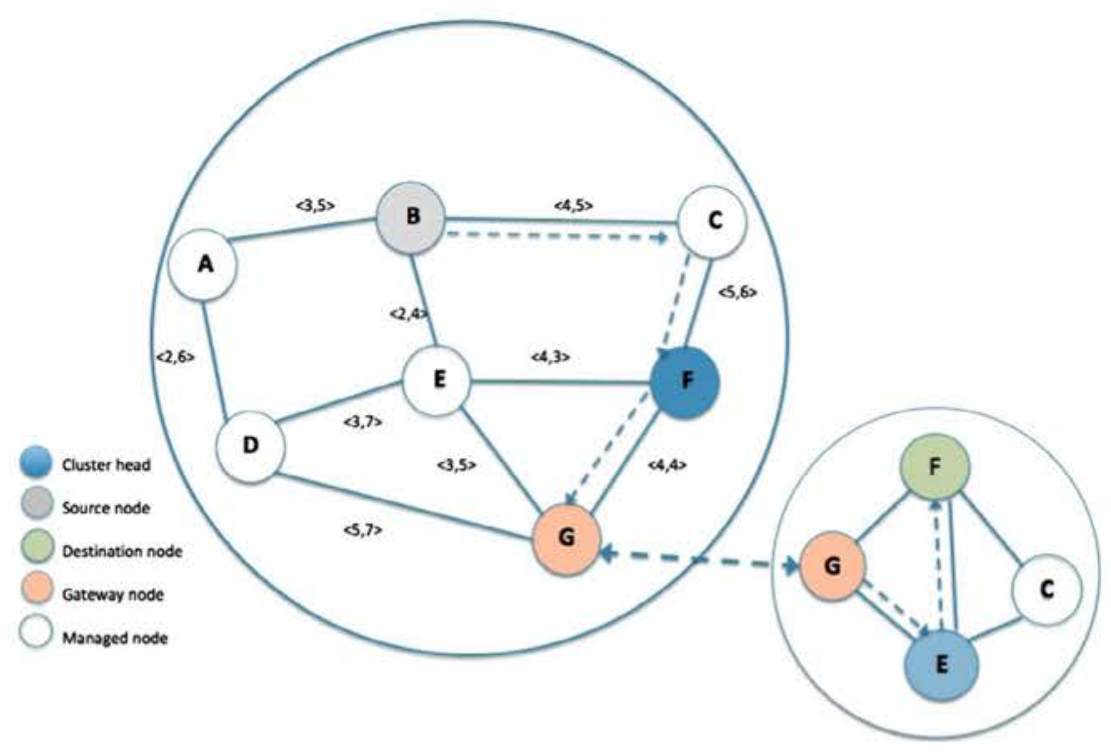

Fig. 1. Clustered ad hoc network.

application of these models will expand in the future (Camp et al., 2002; Niewiadomska-Szynkiewicz et al., 2013). Models of synthetic mobility are already used together with simulated scenarios. In order to prove this form of controlled mobility, certain parameters are used to obtain quantifiable data and, thus, to transform them into useful information. The synthetic models are classified according to their relationship with the representation of human displacement: Unrealistic Synthetic Mobility Models, such as: Random Models (Divecha, Abraham, Grosan, \& Sanyal) (Random Walk Mobility Model, Random Waypoint Mobility Model), Temporal Dependency Models (Hong, Gerla, Pei, \& Chiang, 1999; Divecha et al.) (Boundless Simulation Area Mobility Model, Gauss-Markov Mobility Model, Smooth Random Mobility Model); and Realistic Synthetic Mobility Models, such as: Spatial Dependence Models (Chenchen, Xiaohong, \& Dafang, 2010; Chenchen et al., 2010) (Reference Point Group Mobility, Column Mobility Model, Pursue Mobility Model, Nomadic Mobility Model) and Geographic Restriction Models (Chenchen et al., 2010; Aschenbruck, Gerhards-Padilla, \& Martini, 2008) (Pathway Models, Obstacle Models, Human Obstacle Mobility Model). The three routing protocols that are discussed in this paper: AODV, DSDV and CBRP, used the Random Waypoint Mobility Model in the simulation process, as this is the most related one to human movement. This mobility model is included at the beginning of the code line, inside the connections file that is generated with the NS2 tool.

\section{Proposed scenario:case studies}

As above mentioned, location awareness is an important aspect in the ERS. The success of rescue operations depends on first responders tracking, in-field injured persons triaging, and physical environment monitoring. During emergency situations, lack of experience, complex environments and equipment, or the unawareness of context conditions, affect the classical perception schemes and very often fail or are considerably unsuccessful for saving peoples life. This is why, the analysis of ERS, through simulation of routing protocols, gives us the opportunity to choose the best performing features for them. Before entering the simulation process, it is essential to determine the density of the nodes. In the next section, this calculation is done according to the flow of people in the area. This result is the basic input to the simulation equations.

\subsection{Emergency and rescue scenario for center of the city of Loja}

In order to set the scene for the ERS, Fig. 2, an area of $1000 \mathrm{~m}$ $x 500 m$ in the city Loja was used. The participants urban parishes of the city are: Valle, San Sebastian, Sagrario and Sucre. Random Waypoint Mobility Model have been defined in this area, Figs. 46 that disrupt normal mobility pathways of the nodes.

For each routing protocol and based on figures Figs. 4-6, we will explain the behavior of a node (people with a mobile device) in the disaster area. To explain the movement of the elements in these figures, we will detail the graphical representation of each one of them: Node or person with a mobile device (cyan circle), ${ }^{1}$ goal or evacuation point (red star), and obstacles (red circles with a black stripe in the middle).

For example, if we consider that we are using the DSDV routing protocol in the simulated sector, a node must find the quickest way out of the disaster area and constantly update the information in its routing table. But if many nodes are connected, updating their tables, and occupying a given bandwidth, other nodes may not communicate. Also due to constant updating process, the delay may increase and therefore not allow a quickly departure from the affected area. It is also important to mention that in this type of routing, each node has an information table where you have to update the list of routes, the number of hops to the destination, and the order number assigned by this destination.

On the other side, if we use AODV routing protocol in the simulated area, a node should wait to receive a signal from the target point and then start looking for new communication in the affected location. Internally, a node can also initiate communication to other nodes. Evacuation routes are only given when there is a request. This type of routing in emergency and rescue scenarios may be suitable as long as there are no obstacles in the path. Although each node has two routing tables: an optimized list of routes and the destination sequence number, the obstacles can be a problem. If there is no updated information, communication may be directed to places where there are obstacles and the evacuation time could be higher. Of course, compared to proactive routing, reactive routing is still the best in performance metrics.

\footnotetext{
${ }^{1}$ For interpretation of color in Figs. 5 and 6 , the reader is referred to the web version of this article.
} 


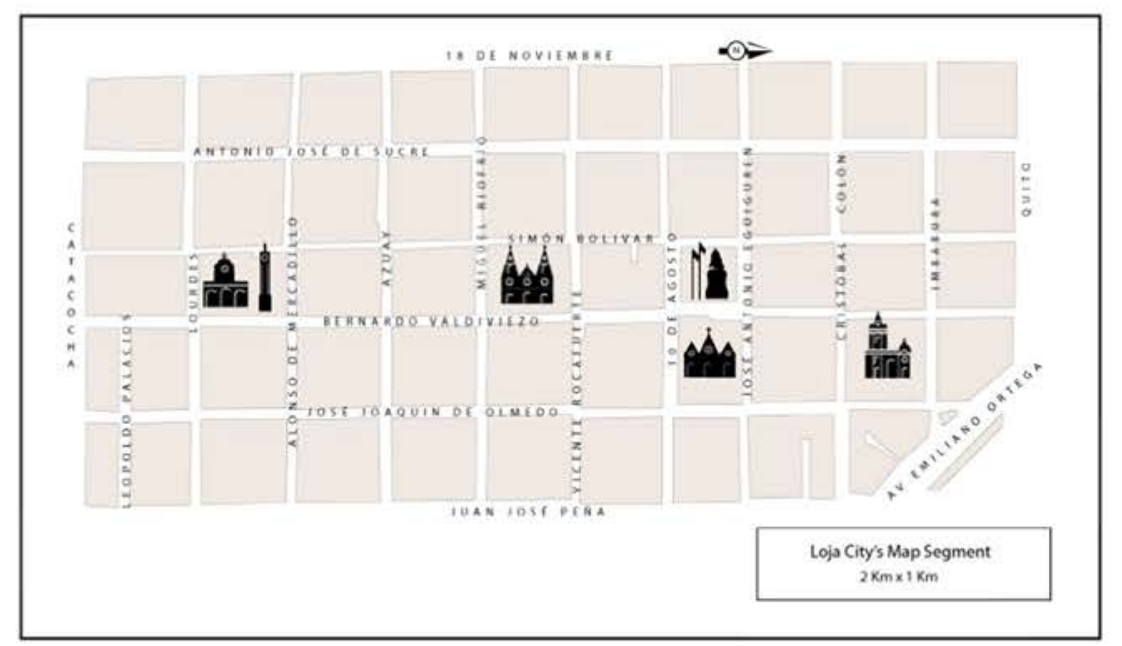

Fig. 2. Action area over Loja city map.

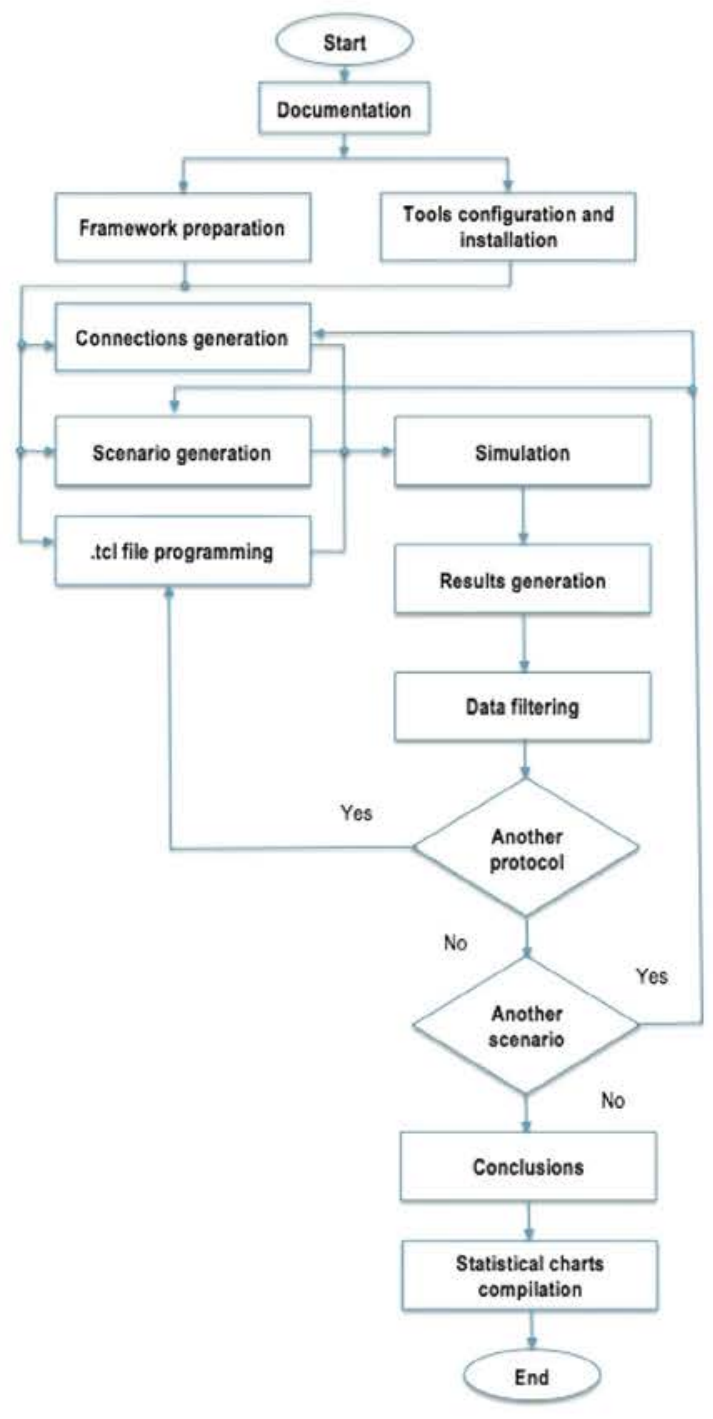

Fig. 3. Methodology for comparative analysis of AODV, DSDV and CBRP protocols.

In contrast, if we use the hierarchical routing protocol, the big picture gets better. Here when a node wants to leave the disaster area, it joins a group and the group designate a leader, which has the ability to get them out faster to the target point. Also while the group leader is fulfilling this task, another node acts as a communicator with nearby groups in order to inform the best point of evacuation. It is important to also note that for this type of routing, each node has a table with information about their neighbors or nodes that are more close to him. This gives it the advantage of being able to rely on them to get out faster from the disaster area. For performance measures, this routing type has shown the best results, as we will see later.

\subsection{Methodology for comparative analysis of routing protocols}

To make a comparative analysis of routing protocols, involved in this case study, it is necessary to establish and consummate a logical sequence of steps, illustrated in Fig. 3. The results obtained, following this methodological process, will be adequate and valid. The development of this analysis can be roughly divided into four phases: preparation, data collection, analysis of results and conclusions. Because of the generality of these stages, they can be decomposed in different subtasks, such as the connections generation or the scenario generation.

\subsection{Node density}

The calculation of the node density $P_{\text {node }}$ is supported by information obtained from the Ecuadorian census in $2010 .^{2}$ An important factor for the calculation of the node density is the percentage of the Economically Active Population (PEA). To calculate the density of nodes the following equation has been proposed:

$P_{\text {nodes }}=\frac{z u_{1} * F_{u e l} * F_{P E A} * F_{u s}}{A_{z u}} * A_{s}$

$P_{\text {nodes }}=97$

$P_{\text {nodes }_{1}}=97$

where:

- $z u_{l}$ - Number of people in the urban areas is 70\% (128910)

- $z r_{l}$ - Number of people in the rural areas is 30\% (85940).

- $F_{u e l}$ - Urban factor specified for the simulation area

- $A_{z} u$ - Urban area $-6 \times 12 \mathrm{Km}=72 \mathrm{~km}^{2}$.

\footnotetext{
${ }^{2}$ National Institute of Statistics and Censuses (INEC). http://www.inec.gob.ec.
} 


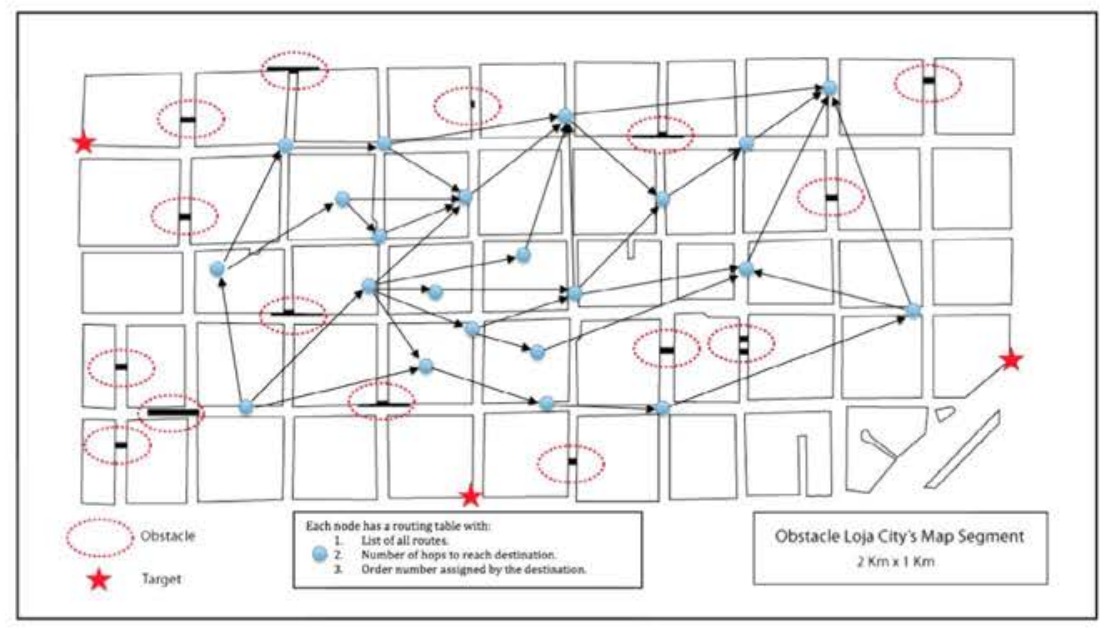

Fig. 4. DSDV routing in the simulated area.

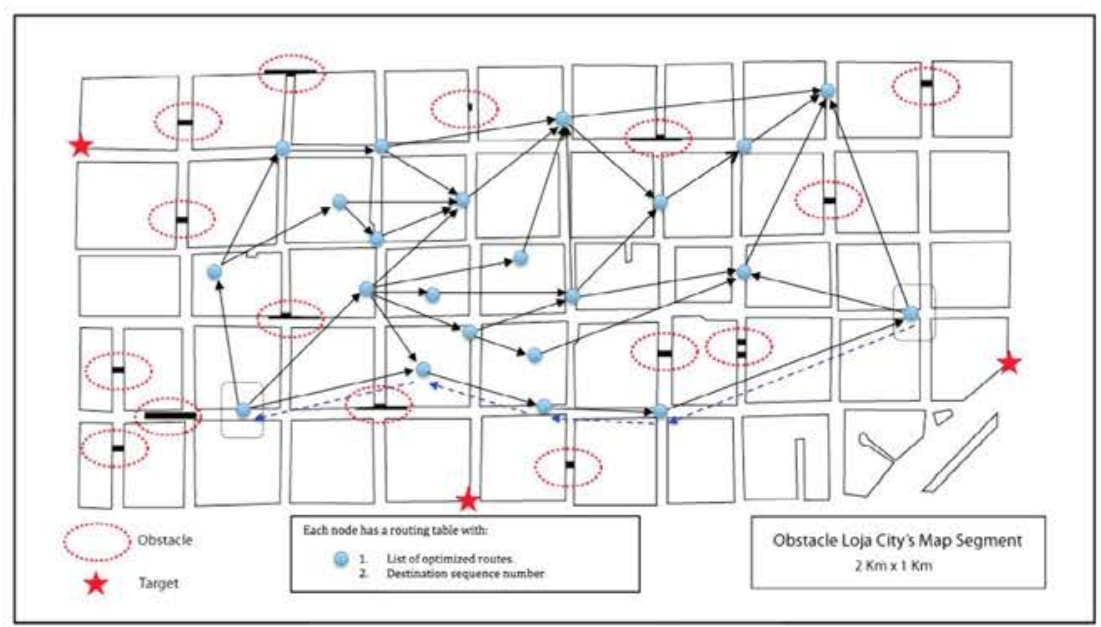

Fig. 5. AODV routing in the simulated area.

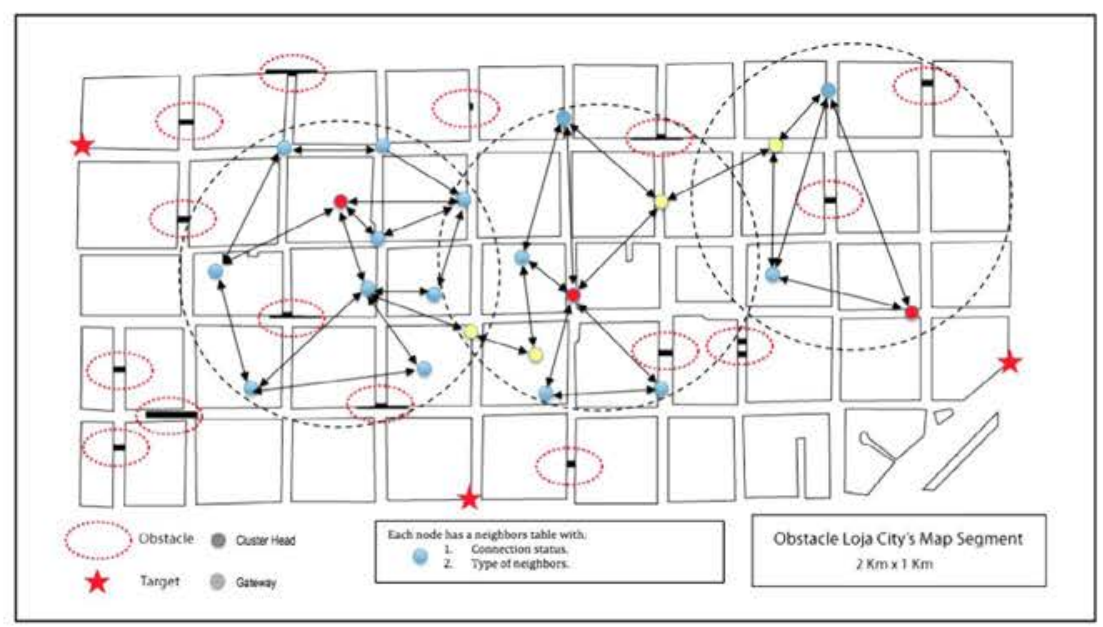

Fig. 6. CBRP routing in the simulated area.

- $F_{P E A}$ - Loja - PEA is $62 \%$ for urban area.

- $F_{u s}$ - Urban smartphone factor is $25 \%$.

- $A_{s}$ - Chosen area for simulation $0.5 \mathrm{~km}^{2}$.

To determine the sample required, it is important to describe the city for which this study has been done; the city is located in the southern part of Ecuador. Population increases during the holidays, and it grows also due to arriving of tourists attracted by its location and biodiversity. Given this premise, we established percentages of $25.75 \%\left(F_{u s}\right), 30.8 \%\left(F_{u s}\right)$ and $34.4 \%\left(F_{u s}\right)$ to calculate the other three node densities $P_{\text {nodes }}$, by substituting these values into the Eq. (1), and considering the mentioned increment of 
people, we found that $P_{\text {nodes }_{2}}, P_{\text {nodes }_{3}}, P_{\text {nodes }_{4}}$ are 100 (25.75\%), 120 $(30.8 \%)$ and 160 (34.4\%), respectively.

Consequently, the node densities for simulation are: $P_{\text {nodes }_{1}}=97$, $P_{\text {nodes }_{2}}=100, P_{\text {nodes }_{3}}=120$ and $P_{\text {nodes }_{4}}=160$.

\subsection{General parameters for simulation}

To define the simulation scenarios we used as the basis (Kurkowski, Navidi, \& Camp, 2007a, 2007b). The values of each one of these values are shown in the Table 1. In order to analyze results, some authors have observed a set of indicators. For our research, and in order to measure behavior of protocols, we selected some, from (Chenna Reddy \& ChandraSekhar Reddy, 2006). These indicators are: performance, protocol overhead, packet dropped, average delay and the variation of the delay or jitter. These indicators are compared with those of the following protocols: CBRP (Chaba et al., 2009), AODV (Shukla et al., 2013) and DSDV (Mahdipour et al., 2009).

\section{Simulation results}

\subsection{Creating scenarios}

We entered via a terminal the following directory: ns-allinone then we entered ns2.34/indeputils/cmugen/setdest/setdest in order to create a scenario of 160 nodes with the following command./set- dest-v 160-2-n-m 2 s 1 m 6-t-150-P-1-p 2 x 2000and 1000 . The sample was successfully created and named Scenario1. This should be replicated to each type of scenario we need to create. In the command shown: run-v 2, which is the scenario generator version; we identify: $n$, which means the number of nodes; $s$, is the kind of speed ( $1=$ Uniform); minspeed, represented with lowercase $\mathrm{m}$, is the minimum speed in $\mathrm{m} / \mathrm{s}$; maxspeed, represented with uppercase $m$, is the maximum speed in $m / s ;-t$, indicating the simulation time in seconds; -P is the pause time simulation in seconds; $\operatorname{maxX}$, the size in meters for the $\mathrm{X}$ dimension; maxY, the size in meters for the $\mathrm{Y}$ dimension; and finally [out$\operatorname{dir}=$ file], Filename motion itself scenario name. The directory should be accessed to verify the creation of the corresponding scenarios.

\subsection{Creating connections}

First, we entered ns-allinone, then, the next route is ns2.34/ indeputils/cmugen/setdest/. In this direction, there must be the cbrgen.tcl file, this file serves me as a basis to create the connections, it should be emphasized that for this type scenarios we created two types of connections 20 and 40 for the amount of 97,100 , 120 and 160 nodes. To generate the connections in this specific case study we accessed via a terminal ns2.34 and executed the following command to create the connections, ns cbrgen.tcl-type tcp-nn 160 seed-mc 40-rate rate 2 > scenconextcp40. Where:
Table 2

Variables and parameters for the simulation of protocols AODV, DSDV and CBRP.

\begin{tabular}{|c|c|c|}
\hline Variable & Value & Observations \\
\hline Set val (chan) & Channel/WirelessChannel & Channel Type \\
\hline \multirow[t]{2}{*}{ Set val (prop) } & Propagation/ & radio-propagation \\
\hline & TwoRayGround & model \\
\hline Set val (netif) & Phy/WirelessPhy & Network interface type \\
\hline Set val (mac) & $\mathrm{Mac} / 802_{1} 1$ & MAC type \\
\hline Set val (ifq) & Queue/DropTail/PriQueue & Interface queue type \\
\hline Set val (II) & $\mathrm{LL}$ & link layer type \\
\hline Set val (ant) & Antenna/OmniAntenna & Antenna model \\
\hline Set val (ifqlen) & 100 & Max packet in ifq \\
\hline Set val (nn) & 156 & Number of mobile nodes \\
\hline Set val (rp) & AODV & Routing protocol \\
\hline Set val (rp) & DSDV & Routing protocol \\
\hline Set val (rp) & CBRP & Routing protocol \\
\hline Set val $(x)$ & 2000 & Area \\
\hline Set val $(y)$ & 1000 & Area \\
\hline $\begin{array}{l}\text { Set val } \\
\quad \text { (conexiones) }\end{array}$ & "scenconextcp $40 "$ & Connections \\
\hline Set val (scenario) & "scen120total" & Scenario \\
\hline Set val (stop) & 150 & Time simulation \\
\hline
\end{tabular}

tcp-, is the type of traffic; nn-, indicates the number of nodes in the simulation; seed, random seed; mc-, connections number of connections; -rate, indicates the baud rate in pkts/s; and finally [outdir $=$ file] that becomes the file name connections.

\subsection{Performance metrics}

The NS2 simulator (NS2) is used to determine the protocols behavior with the data shown in Table 2 . In order to determine which is the best protocol, we used the following indicators for comparison:

- Average delay. This is very significant to measure for our purpose, because there is a need to send and receive network management information as fast as possible. In this parameter, as shown in Fig. 7, with 20 connections and with 97 nodes, the 3 protocols behave according to their characteristics. But, with 100,120 and 160 nodes, CBRP protocol suffers small delays. Instead with 40 connections as seen in Fig. 8, the panorama changes to demonstrate advantages that protocol CBRP has over AODV and DSDV protocols, for managing numerous nodes and connections. Data are included in Tables 3 and 4.

- Packet delay variation - It is the difference or delay between end-to-end communication selected packets. It serves to measure the network stability and convergence, in MANETs. This parameter is related to the mean fluctuation and helps us to determine which of the three protocols would be the most appropriate at the time of an emergency or rescue. For CBRP protocol, this jitter parameter is near to zero as seen in Figs. 9 and 10 . The simulation results are set to Tables 5 and 6 .

Table 1

General parameters for the simulation.

\begin{tabular}{|c|c|c|}
\hline Parameter & Value & Observations and related works \\
\hline Simulation area & $1000 \times 2000 \mathrm{~m}$ & Area established within the center of the city of Loja \\
\hline Number of nodes & $97,100,120$ and 160 & To determine the behavior of protocols for different node density \\
\hline Number of connections & 20,40 & random connections \\
\hline Time of simulation & 150 & In seconds \\
\hline Network layer protocols & CBRP, DSDV and AODV & Hierarchical, proactive and reactive protocols \\
\hline Transport layer protocols & Transmission control protocol (TCP) & A connection-oriented communication is needed \\
\hline Propagation model & TwoRayGround & For flat and unobstructed scenarios. \\
\hline Type of antenna & Omnidirectional & Indispensable quality of mobile network nodes \\
\hline
\end{tabular}




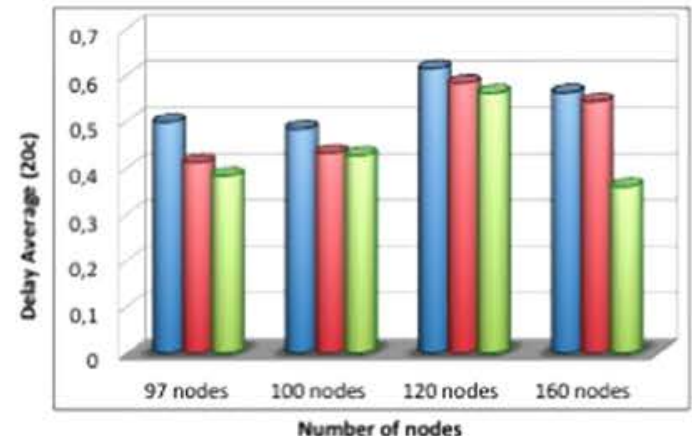

Fig. 7. Delay Average - TCP-20 connections.

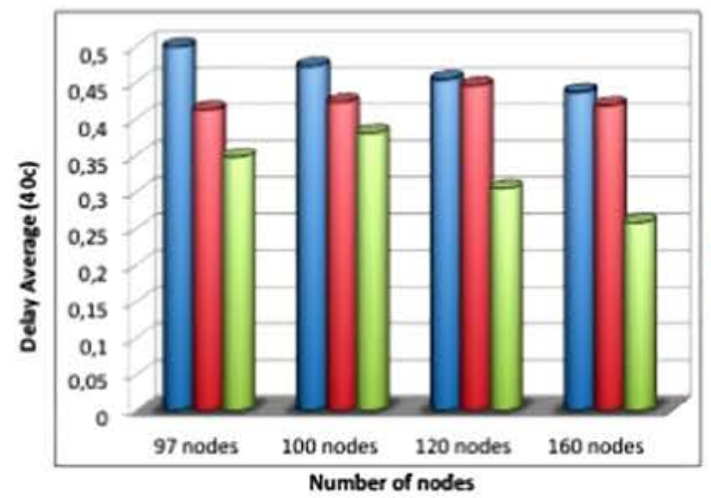

Fig. 8. Delay Average - TCP-40 connections.

Table 3

AODV, DSDV, CBRP protocols analysis - Delay Average 20 connections.

\begin{tabular}{llll}
\hline Number of nodes & AODV (20c) & DSDV (20c) & CBRP (20c) \\
\hline 97 & 0.498009 & 0.411367 & 0.383271 \\
100 & 0.483952 & 0.432102 & 0.427227 \\
120 & 0.613480 & 0.582582 & 0.560971 \\
160 & 0.561865 & 0.543427 & 0.359856 \\
\hline
\end{tabular}

Table 4

AODV, DSDV, CBRP protocols analysis - Delay Average 40 connections.

\begin{tabular}{llll}
\hline Number of nodes & AODV $(40 \mathrm{c})$ & DSDV $(40 \mathrm{c})$ & CBRP $(40 \mathrm{c})$ \\
\hline 97 & 0.498009 & 0.411367 & 0.346918 \\
100 & 0.471993 & 0.421802 & 0.379617 \\
120 & 0.452916 & 0.444208 & 0.303840 \\
160 & 0.435439 & 0.417546 & 0.257654 \\
\hline
\end{tabular}

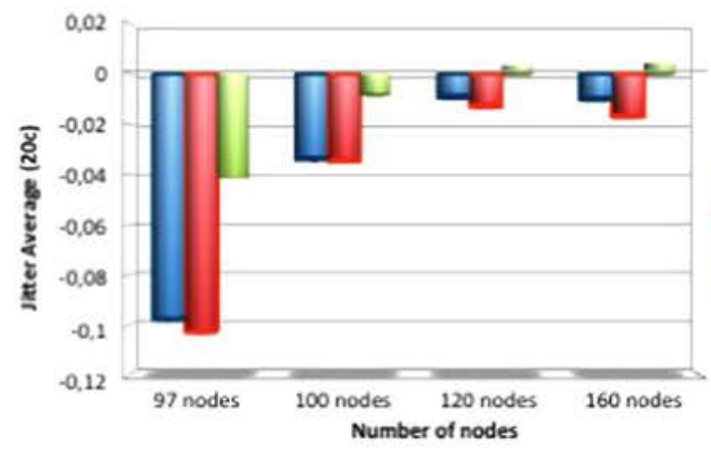

Fig. 9. Jitter Average TCP 20 connections.

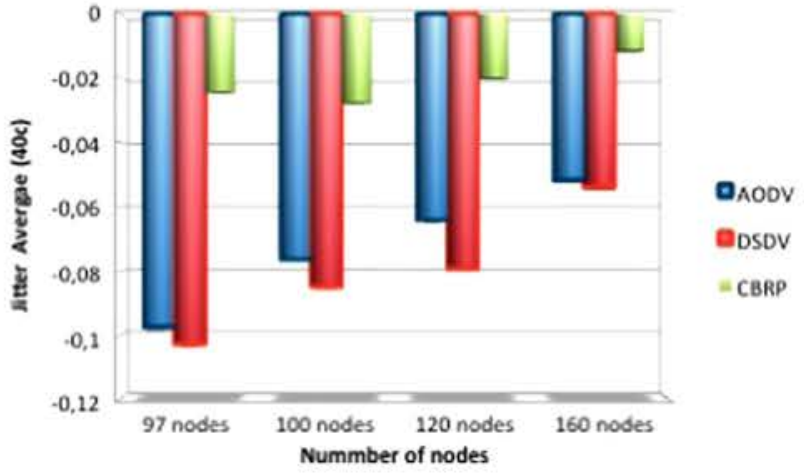

Fig. 10. Jitter Average TCP 40 connections.

Table 5

AODV, DSDV, CBRP protocols analysis - Jitter Average 20 connections.

\begin{tabular}{lccr}
\hline Number of nodes & AODV $(20 \mathrm{c})$ & DSDV $(20 \mathrm{c})$ & CBRP $(20 \mathrm{c})$ \\
\hline 97 & -0.097499 & -0.102806 & -0.041516 \\
100 & -0.033695 & -0.034596 & -0.008314 \\
120 & -0.009072 & -0.012740 & 0.003136 \\
160 & -0.009954 & -0.016830 & 0.004321 \\
\hline
\end{tabular}

Table 6

AODV, DSDV, CBRP protocols analysis - Jitter Average 40 connections.

\begin{tabular}{llll}
\hline Number of nodes & AODV (40c) & DSDV (40c) & CBRP (40c) \\
\hline 97 & -0.097499 & -0.102806 & -0.024319 \\
100 & -0.076439 & -0.085109 & -0.027638 \\
120 & -0.064236 & -0.079349 & -0.019921 \\
160 & -0.051754 & -0.053986 & -0.011346 \\
\hline
\end{tabular}

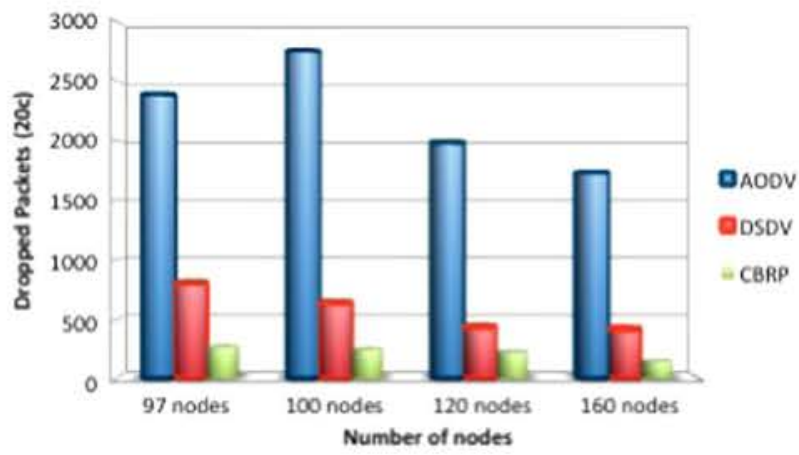

Fig. 11. Dropped packets - 20 connections.

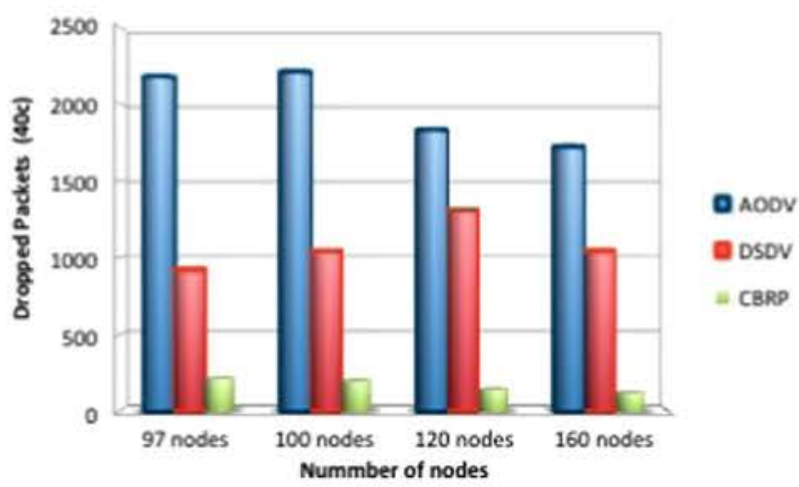

Fig. 12. Dropped packets - 40 connections. 
Table 7

AODV, DSDV, CBRP protocols analysis - Dropped Packets - 20 connections.

\begin{tabular}{llll}
\hline Number of nodes & AODV (20c) & DSDV (20c) & CBRP (20c) \\
\hline 97 & 2387 & 799 & 269 \\
100 & 2753 & 631 & 245 \\
120 & 1984 & 429 & 222 \\
160 & 1732 & 412 & 142 \\
\hline
\end{tabular}

Table 8

AODV, DSDV, CBRP protocols analysis - Dropped Packets - 40 connections.

\begin{tabular}{llcl}
\hline Number of nodes & AODV $(40 \mathrm{c})$ & DSDV $(40 \mathrm{c})$ & CBRP $(40 \mathrm{c})$ \\
\hline 97 & 2175 & 921 & 212 \\
100 & 2206 & 1041 & 196 \\
120 & 1830 & 1310 & 142 \\
160 & 1721 & 1043 & 117 \\
\hline
\end{tabular}

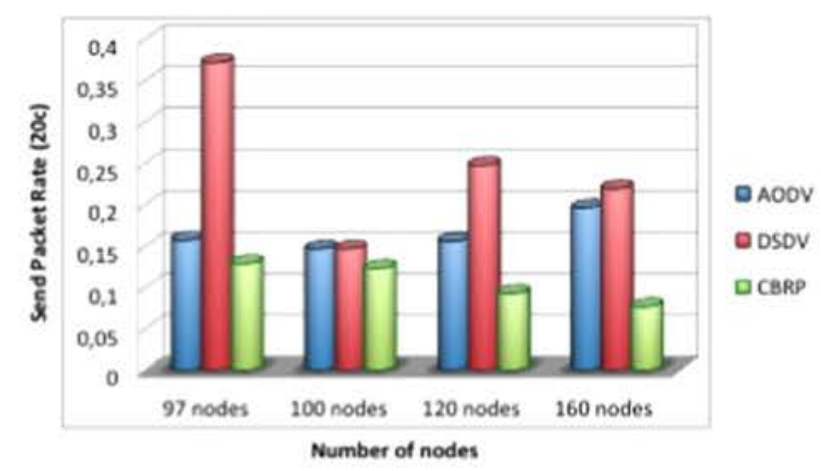

Fig. 13. Send Packet Rate 20 connections.

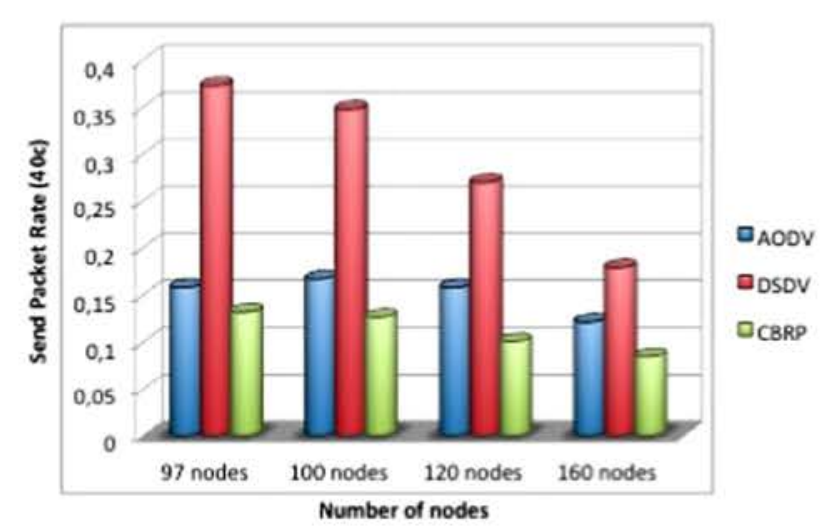

Fig. 14. Send Packet Rate 40 connections.

Table 9

AODV, DSDV, CBRP protocols analysis - Send Packet Rate 20 connections - Send All/ Rec All.

\begin{tabular}{llll}
\hline Number of nodes & AODV (20c) & DSDV (20c) & CBRP (20c) \\
\hline 97 & 0.157762 & 0.371905 & 0.129553 \\
100 & 0.147154 & 0.305931 & 0.123468 \\
120 & 0.156513 & 0.247538 & 0.092591 \\
160 & 0.1965432 & 0.21965 & 0.07654 \\
\hline
\end{tabular}

- Packet Dropped - This is the amount of packets dropped by intermediate nodes due to the effects produced by its own mobility, the expiration of time, and unreachable or erased destinations by Address Resolution Protocol (ARP).
Table 10

AODV, DSDV, CBRP protocols analysis - Send Packet Rate 40 connections - Send All] Rec All.

\begin{tabular}{llll}
\hline Number of nodes & AODV (40c) & DSDV (40c) & CBRP (40c) \\
\hline 97 & 0.157762 & 0.371905 & 0.131649 \\
100 & 0.166630 & 0.346906 & 0.125966 \\
120 & 0.157641 & 0.269684 & 0.100301 \\
160 & 0.120956 & 0.178650 & 0.084326 \\
\hline
\end{tabular}

The objective of the simulation was to determine which of these three protocols behave better in ERS and how we can see in Figs. 11 and 12 CBRP protocol clears least packets with 20 connections and even better with 40 connections. The data are shown in Tables 7 and 8.

- Packets sent rate - The rate obtained by the number of packets sent versus the number of packets received. For formation and maintenance, the cluster needs the exchange of packets for having updated information. As shown in Figs. 13 and 14, the protocol that best responds to this parameter is the CBRP, for both 20 and 40 connections. The data are shown in Tables 9 and 10 .

\section{Conclusions}

- Traditional routing algorithms cannot satisfy requirements of a MANET, because of the topology dynamics and limited bandwidth characterizing these networks. Consequently, there is a lot of research related to existing routing algorithms, but there is also room for discovering new routing algorithms, which are more efficient.

- This study evaluates and compares CBRP, AODV and DSDV protocols for ERS. The experimental results show that the best protocol for these cases is CBRP. CBRP looses little information during the routing processes, and its sending and receiving rate of packets is stable. The mean fluctuation and the delay are also much smaller in CBRP than in AODV and DSDV. Finally, this research suggests that the use of CBRP protocol in a disaster area, more efficiently adjusts the evacuation of persons and their care and appropriate location.

\section{Acknowledgements}

The authors of this paper thank the Research Institute of Computer Science, Technical University of Loja, and the Department of Computing, Polytechnic University of Madrid, for providing the necessary resources to complete this research. Special thanks go to B.S. Jorge Jaramillo E. for his able research assistance.

\section{References}

Ali, N. Z., Ahmad, R. B., \& Aljunid, S. A. (2008). Link availability estimation for routing metrics in MANETs: An overview. Time, 6-8.

Aschenbruck, N., Gerhards-Padilla, E., \& Martini, P. (2008). A survey on mobility models for performance analysis in tactical mobile networks.

Biradar, R., \& Patil, V. (2006). Classification and comparison of routing techniques in wireless ad hoc networks. In International symposium on ad hoc and ubiquitous computing, ISAUHC '06 (pp. 7-12).

Camp, T., Boleng, J., \& Davies, V. (2002). A survey of mobility models for ad hoc network research. Wireless Communications and Mobile Computing. Special issue on Mobile Ad Hoc Networking. Research, Trends and Applications, 2, 483-502.

Chaba, Y., Singh, Y., \& Joon, M. (2009). Performance evaluation and analysis of cluster based routing protocols in manets. 64-66. [cited By (since 1996) 3].

Chenchen, Y., Xiaohong, L., \& Dafang, Z. (2010). An obstacle avoidance mobility model. In 2010 IEEE International Conference on Intelligent Computing and Intelligent Systems (ICIS) (Vol. 3, pp. 130-134).

Chenna Reddy, P., \& ChandraSekhar Reddy, P. (2006). Performance analysis of adhoc network routing protocols. In International symposium on ad hoc and ubiquitous computing, ISAUHC 'O6 (pp. $186-187$ ).

Chen, A. Y., Pea-Mora, F., Plans, A. P., Mehta, S. J., \& Aziz, Z. (2012). Supporting urban search and rescue with digital assessments of structures and requests of 
response resources. Advanced Engineering Informatics. SI: Modern Concurrent Engineering, 26(4), 833-845.

Divecha, B., Abraham, A., Grosan, C., \& Sanyal, S. Impact of node mobility on manet routing protocols models.

Er, 1., \& Seah, W. (2005). Clustering overhead and convergence time analysis of the mobility-based multi-hop clustering algorithm for mobile ad hoc networks. In 11 th international conference on parallel and distributed systems, Proceedings (Vol. 2, pp. 130-134).

Er, 1., \& Seah, W. (2004). Mobility-based d-hop clustering algorithm for mobile ad hoc networks. In Wireless communications and networking conference, WCNC (Vol. 4, pp. 2359-2364). IEEE.

Er, 1., \& Seah, W. (2004). Mobility-based d-hop clustering algorithm for mobile ad hoc networks. In Wireless communications and networking conference, WCNC (Vol. 4, pp. 2359-2364). IEEE.

Fujiwara, T., \& Watanabe, T. (2005). An ad hoc networking scheme in hybrid networks for emergency communications. Ad Hoc Networks, 3(5), 607-620.

Gupta, S. K., \& Saket, R. (2011). Performance metric comparison of aodv and dsdv routing protocols in manets using ns-2. IJRRAS, 7(3), 339-350.

Hong, X., Gerla, M., Pei, G., \& Chiang, C.-C. (1999). A group mobility model for ad hoc wireless networks. In Proceedings of the 2nd ACM international workshop on modeling, analysis and simulation of wireless and mobile systems, MSWiM' 99 (pp. 53-60). New York, NY, USA: ACM.

Jadeja, N., \& Patel, R. (2013). Performance evaluation of aodv, dsdv and dsr routing protocols using ns-2 simulator. Performance Evaluation, 3(2), :1825-1830.

Jahani, S., \& Bagherpour, M. (2011). A clustering algorithm for mobile ad hoc networks based on spatial auto-correlation. In 2011 International symposium on computer networks and distributed systems (CNDS) (pp. 136-141).

Johnson, D., Hu, Y., \& Maltz, D. (2007). The dynamic source routing protocol (DSR) for mobile ad hoc networks for IPv4. Number 4728 in Request for Comments. Internet Engineering Task Force, IETF.

Kumawat, R., \& Somani, V. (2011). Article: Comparative analysis of dsdv and olst routing protocols in manet at different traffic load. In IJCA Proceedings on Intemational Conference on Computer Communication and Networks CSI-COMNET2011. Comnet (Vol. 1). New York, USA: Foundation of Computer Science.

Kurkowski, S., Navidi, W., \& Camp, T. (2007). Constructing manet simulation scenarios that meet standards. In IEEE internatonal conference on mobile adhoc and sensor systems, MASS 2007 (pp. 1-9).

Kurkowski, S., Navidi, W., \& Camp, T. (2007b). Discovering variables that affect manet protocol performance. In Global telecommunications conference, GLOBECOM '07 (pp. 1237-1242). IEEE.

Mahdipour, E., Rahmani, A. M., \& Aminian, E. (2009). Performance evaluation of destination-sequenced distance-vector (dsdv) routing protocol. International Conference on Future Networks, 0, 186-190.
Mahesh Motwani, Y., \& Agarwal, R. (2009). Survey of clustering algorithms for manet.

McDonald, A., \& Znati, T. (1999). A mobility-based framework for adaptive clustering in wireless ad hoc networks. IEEE Journal on Selected Areas in Communications, 17(8), 1466-1487.

Niewiadomska-Szynkiewicz, E., Sikora, A., \& KoAodziej, J. (2013). Modeling mobility in cooperative ad hoc networks. Mobile Networks and Applications, 1-12.

Ns. The Network Simulator NS-2. <http://www.isi.edu/nsnam/ns/>.

Overview, R.-A., \& Selangor, U. P. M. S. (2007). Existing MANET routing protocols and metrics used towards the efficiency and reliability-an overview. IEEE, 231-236 [Number May].

Ramrekha, T., Talooki, V., Rodriguez. J., \& Politis, C. (2012). Energy efficient and scalable routing protocol for extreme emergency ad hoc communications. Mobile Networks and Applications, 17(2), 312-324.

Reina, D. G., Toral, S., Barrero, F., Bessis, N., \& Asimakopoulou, E. (2011). Evaluation of ad hoc networks in disaster scenarios. In 2011 third international conference on intelligent networking and collaborative systems (INCOS) (pp. 759-764).

Reina, D., Marn, S. T., Bessis, N., Barrero, F., \& Asimakopoulou, E. (2013). An evolutionary computation approach for optimizing connectivity in disaster response scenarios. Applied Soft Computing, 13(2), 833-845.

Reina, D., Toral, S., Barrero, F., Bessis, N., \& Asimakopoulou, E. (2012). Modelling and assessing ad hoc networks in disaster scenarios. Journal of Ambient Intelligence and Humanized Computing, 1-9.

Sadasivam, K., Changrani, V., \& Yang, T. A. (2005). Scenario based performance evaluation of secure routing in manets. In L. T. Yang, H. R. Arabnia, \& L.-C. Wang (Eds.), ICWN (pp, 24-32), CSREA Press.

Shukla, A., Jha, C., Saxena, N., \& Biswash, S. (2013). The analysis of aodv, based on mobility model. (pp. 440-443). [cited By (since 1996) 0].

Thorsten Aurisch, J. T. (2009). Relay placement for ad-hoc networks in crisis and emergency scenarios. In NATO,OTAN (pp. 142-152).

Torres, R., Mengual, L., Marban, O., Eibe, S., Menasalvas, E., \& Maza, B. (2012). A management ad hoc networks model for rescue and emergency scenarios. Expert Systems with Applications, 39(10), 9554-9563 [cited By (since 1996) 3]..

Ugas, J.C. (2009). Estudio y analisis de prestaciones de redes mviles ad hoc mediante simulaciones ns-2 para validar modelos analticos. In Tesis doctoral- universidad politcrica de catalua (pp. 25 - 26).

Zang, C. \& Tao, C. (2009). A k-hop passive cluster based routing protocol for manet. In 5 th international conference on wireless communications, networking and mobile computing, WiCom '09 (pp. 1-4).

Zhiyuan, L. \& Jinhong, H. (2010). Simulation and analysis of optimized olsr. In 2010 International conference on multimedia information networking and security (MINES) (pp. 97-100). 